\title{
NUMERICAL ANALYSIS ON DISASTER MITIGATION SEAWALL WITH MOVABLE CROWN USING COUPLED FLUID-STRUCTURE INTERACTION MODEL
}

\author{
Tomoaki NAKAMURA ${ }^{1}$, Yasuo KOTAKE², \\ Akiko MATSUMURA ${ }^{3}$ and Norimi MIZUTANI ${ }^{4}$ \\ ${ }^{1}$ Member of JSCE, Designated Associate Professor, Institute for Advanced Research, Nagoya University \\ (Furo-cho, Chikusa-ku, Nagoya, Aichi 464-8601, Japan) \\ E-mail: tnakamura@nagoya-u.jp \\ ${ }^{2}$ Member of JSCE, Technical Research Institute, Toyo Construction Co., Ltd. \\ (1-25-1, Naruohama, Nishinomiya, Hyogo 663-8142, Japan) \\ E-mail: kotake-yasuo@toyo-const.co.jp \\ ${ }^{3}$ Technical Research Institute, Toyo Construction Co., Ltd. \\ (1-25-1, Naruohama, Nishinomiya, Hyogo 663-8142, Japan) \\ E-mail: matsumura-akiko@toyo-const.co.jp \\ ${ }^{4}$ Member of JSCE, Professor, Department of Civil Engineering, Nagoya University \\ (Furo-cho, Chikusa-ku, Nagoya, Aichi 464-8603, Japan) \\ E-mail:mizutani@civil.nagoya-u.ac.jp
}

\begin{abstract}
A coupled fluid-structure-sediment interaction model is applied to a tsunami/storm-surge disaster mitigation structure attached with a movable crown to investigate the mechanisms of the movable crown behavior and wave energy dissipation. The model is composed of a large-eddy simulation solver for computing incompressible viscous air-water flow that considers the motion of a movable structure and the profile evolution of the seabed, a volume-of-fluid module for tracking air-water interface motion, an immersedboundary module for computing movable structure motion, and a sediment transport module for computing suspended sediment motion and seabed profile evolution. The modules are connected to the solver using a two-way coupling procedure to ensure fluid-structure-sediment interaction. Numerical results show that the relation between the fluid inflow/outflow rate through a gap in the crown and the angle of the movable crown is an important parameter in predicting the wave field around the structure and the behavior of the movable crown. This suggests that it is essential to calibrate the parameter against the experimental data in numerically evaluating the performance of the structure for optimal design. It is also found that the motion of the movable crown is affected by the air trapped in the flood-control basin at the time of uprush and the air flow entering the flood-control basin at the time of backwash, suggesting that it is important to consider not only water flow but also air flow in the numerical simulation. Furthermore, the structure is found to efficiently dissipate wave energy due to vortices forming around the crown and the motion of the movable crown.
\end{abstract}

Key Words : disaster mitigation structure, movable crown, numerical analysis, fluid-structure interaction, wave energy dissipation

\section{INTRODUCTION}

When a structure faces high wind waves, tsunamis, or storm surges of a magnitude beyond the level predicted at the time of its design, it is difficult to fully protect the area behind the structure from inundation damage due to wave overtopping or wave overflow $^{1)}$. Accordingly, it is necessary to develop non-structural measures in collaboration with disas- ter mitigation structures. Efficient and effective maintenance according to life-cycle management are important for the aging coastal structures built during the period of rapid growth in Japan ${ }^{2)}$, because most of them are deteriorating and in need of renovation. To meet such demands, Kotake et al. ${ }^{3)}$ have proposed a tsunami/storm-surge disaster mitigation structure, which is relatively simple and easy to remodel. Figure 1 shows an overview of this structure. As 


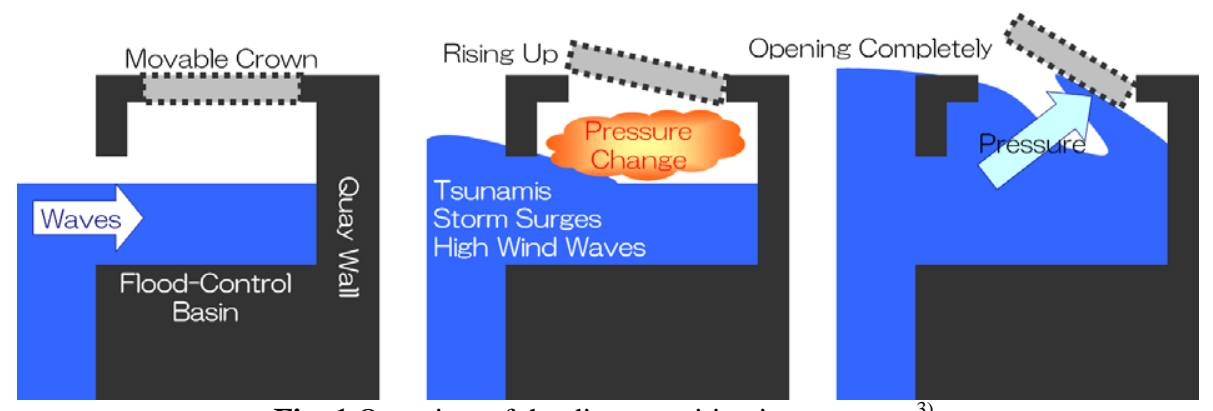

Fig. 1 Overview of the disaster mitigation structure ${ }^{3)}$.

shown, this structure consists of a flood-control ba$\sin$ in front of an existing vertical quay wall and a levee crown that opens and closes with waves (hereafter referred to as a "movable crown") in order to mitigate wave overtopping rates caused by high wind waves, tsunamis, or storm surges, and to delay the onset of inundation in areas behind the structure. In a hydraulic experiment, this mechanism has demonstrated low reflection coefficients and wave overtopping rates for wind waves, and the ability to delay the onset of inundation for tsunamis ${ }^{3)}$. However, the hydraulic experiment only allowed qualitative analyses of the mechanisms of the movable crown behavior and wave energy dissipation associated with the low reflection coefficients because of the lack of experimental data.

In this study, a three-dimensional coupled fluidstructure-sediment interaction model by Nakamura and Mizutani ${ }^{4)}$, which has the computational capability for analyzing air-water interaction as well as air-water-structure interaction, is applied to the hydraulic experiment by Kotake et al. ${ }^{3)}$, because the action of water mass due to waves and the motion of air trapped in the flood-control basin are expected to be essential to the behavior of the movable crown. To verify the usefulness of the model for designing such structures, the predictive capability of the model is verified through comparison with experimental results in terms of water level changes around the structure and the behavior of the movable crown. Furthermore, the mechanisms of the movable crown behavior and wave energy dissipation are analyzed and discussed for more detail.

\section{COUPLED FLUID-STRUCTURE- SEDIMENT INTERACTION MODEL}

The three-dimensional coupled fluid-structuresediment interaction model ${ }^{4)}$ is composed of a main solver and three modules. The main solver is a largeeddy simulation (LES) model based on extended continuity and momentum equations for incompressible viscous air-water flow that considers seepage flow in porous media, the motion of a movable structure, and the profile evolution of the seabed. The first module is a volume-of-fluid (VOF) module based on the multi-interface advection and reconstruction solver (MARS) ${ }^{5}$, which was an improvement of the VOF method, for tracking air-water interface motion. The second module is an immersed-boundary (IB) module based on the volume-force type of IB method ${ }^{6)}$ for computing the motion of the movable structure. The third module is a sediment transport module for computing the seabed profile evolution induced by bed-load and suspended sediment transport and the motion of suspended sediment that considers all transport processes of pickup, advection, diffusion, and settling. The modules are connected to the solver using a two-way coupling procedure to ensure fluid- structure-sediment interaction. In this study, the main solver, the VOF module, and the IB module were employed to compute the wave-induced motion of the movable crown. For completeness, the main solver, the VOF module, and the IB module are briefly presented below. A detailed explanation of the model can be found in Nakamura and Mizutani ${ }^{4)}$. For a numerical analysis of fluid-structure interaction using the model, see Nakamura et al. ${ }^{7}$.

In the VOF method, the VOF function $F$ represents the volume fraction of water in each cell $(0 \leq F$ $\leq 1$, where $F=0$ for pure air, $0<F<1$ for air-water interface, and $F=1$ for pure water). Similarly, a movable structure is represented as the volume fraction of the structure in each cell $F_{o b}\left(0 \leq F_{o b} \leq 1\right.$, where $F_{o b}=0$ outside the structure, $0<F_{o b}<1$ for structure surface, and $F_{o b}=1$ inside the structure) in the volume-force type of IB method ${ }^{6)}$. The fluidstructure interaction force $\mathbf{f}^{o b}$ as a function of $F_{o b}$ is determined in such a way that the volume-average velocity $\mathbf{v}$ inside the structure is equal to the velocity of the structure $\mathbf{v}^{o b}$, and applied to fluids in numerical cells where $F_{o b}>0$. Here, $\mathbf{v}$ is defined as $\left(1-F_{o b}\right) \mathbf{v}^{f}+$ $F_{o b} \mathbf{v}^{o b}\left(\mathbf{v}^{f}=\right.$ fluid/seepage flow velocity vector), and the value of $\mathbf{v}^{o b}$ is calculated from $\mathbf{u}^{o b}+\omega^{o b} \times \mathbf{r}^{o b}\left(\mathbf{u}^{o b}\right.$ and $\boldsymbol{\omega}^{o b}$ are translational and angular velocity vectors of the structure, and $\mathbf{r}^{o b}$ is relative position vector 
from the center of gravity of the structure).

The volume porosity $m$ representing the volume fraction of void space in each cell $(0 \leq m \leq 1$, where $m=0$ for pure impermeable solids, $0<m<1$ for porous media, and $m=1$ for pure fluids) is assumed to be equal to the surface porosity. Furthermore, temporal variation in $m$ is assumed to be sufficiently small. Based on these assumptions, the extended governing equations for continuity, momentum, and air-water interface motion are given as follows:

$$
\begin{gathered}
\frac{\partial\left(m v_{j}\right)}{\partial x_{j}}=q^{*} \\
\left\{m+C_{A}(1-m)\right\} \frac{\partial v_{i}}{\partial t}+\frac{\partial\left(m v_{i} v_{j}\right)}{\partial x_{j}} \\
=-\frac{m}{\hat{\rho}} \frac{\partial p}{\partial x_{i}}+m g_{i}+\frac{m}{\hat{\rho}}\left(f_{i}^{s}+R_{i}+f_{i}^{o b}\right) \\
+\frac{1}{\hat{\rho}} \frac{\partial}{\partial x_{j}}\left(2 m \hat{\mu} D_{i j}\right)+\frac{\partial}{\partial x_{j}}\left(-m \tau_{i j}^{a}\right)+Q_{i}+m \beta_{i} \\
m \frac{\partial F}{\partial t}+\frac{\partial\left(m v_{j} F\right)}{\partial x_{j}}=F q^{*}
\end{gathered}
$$

in which $p=$ pressure, $\mathbf{x}=$ position vector $[x y z]^{\mathrm{T}}, t=$ time, $\mathbf{g}=$ gravitational acceleration vector $\left[\begin{array}{lll}0 & 0 & -g\end{array}\right]^{\mathrm{T}}$ ( $g$ = gravitational acceleration), $\hat{\rho}=F \rho_{w}+(1-F)$ $\rho_{a}=$ density of fluid ( $\rho_{w}$ and $\rho_{a}=$ densities of water and air), $\hat{\mu}=F \mu_{w}+(1-F) \mu_{a}=$ molecular viscosity of fluid ( $\mu_{w}$ and $\mu_{a}=$ molecular viscosities of water and air), $C_{A}=$ added mass coefficient ${ }^{8)}, \mathbf{R}=$ laminar and turbulent resistance force vector due to porous media $^{8)}, \mathbf{f}^{s}=$ surface tension force vector based on the continuum surface force (CSF) model ${ }^{9)}, \tau_{i j}=$ turbulent stress tensor based on the dynamic two-parameter mixed model $(\mathrm{DTM})^{10)}, D_{i j}=\partial v_{i} / \partial x_{j}+\partial v_{j} / \partial x_{i}=$ strain rate tensor, $q^{*}=$ intensity of wave generation source/sink per unit of time ${ }^{11)}, \mathbf{Q}=$ wave generation source/sink vector, $\boldsymbol{\beta}=$ artificial damping factor vector $[00-\beta w]^{\mathrm{T}}$ ( $\beta=$ artificial damping factor), and superscript $a=$ anisotropic part of a tensor. In deriving Eq. (2), the spatial variation in the porosity is taken into account $\left(\partial m / \partial x_{i} \neq 0\right)$ to capture possible sharp changes in the porosity around the surface of the porous media. However, based on the formulation of CADMAS-SURF ${ }^{12}$, the spatial variation in the porosity is assumed to be negligible $\left(\partial m / \partial x_{i}=0\right)$ only in deriving the pressure gradient term of the momentum conservation equation (the first term on the right-hand side of Eq. (2)). This is to ensure equilibrium between the pressure gradient term and the gravitational acceleration term (the second term on the right-hand side of Eq. (2)) in still water regardless of the spatial changes in the porosity. In Eq. (2), $f_{i}^{s}, \tau_{i j}^{a}, R_{i}$, and $Q_{i}$ are given as

$$
\begin{gathered}
f_{i}^{s}=\sigma \kappa \frac{\partial F}{\partial x_{i}} \frac{\hat{\rho}}{\rho_{a v g}} \\
\tau_{i j}^{a}=L_{i j}^{m a}+C_{B} L_{i j}^{R a}-C_{s}|D| D_{i j} \\
R_{i}=-\frac{12 C_{D 2} \hat{\mu}(1-m)}{m d_{50}^{2}} v_{i} \\
-\frac{C_{D 1} \hat{\rho}(1-m)}{2 m d_{50}} v_{i} \sqrt{v_{j} v_{j}} \\
Q_{i}=v_{i} q^{*}-\frac{2}{3} \frac{\partial}{\partial x_{i}}\left(m \hat{v} \frac{\partial v_{j}}{\partial x_{j}}\right)
\end{gathered}
$$

in which $\sigma=$ surface tension coefficient, $\kappa=$ local surface curvature, $\rho_{a v g}=\left(\rho_{w}+\rho_{a}\right) / 2=$ density of fluid at the air-water interface, $C_{D 2}$ and $C_{D 1}=$ laminar and turbulent resistance coefficients ${ }^{8)}, d_{50}=$ median grain size of sediment particles, $\hat{v}=\hat{\mu} / \hat{\rho}=F v_{w}+$ $(1-F) v_{a}=$ kinematic molecular viscosity of fluid ( $v_{w}$ and $v_{a}=$ kinematic molecular viscosities of water and air), $|D|=$ absolute value of the strain rate tensor $D_{i j}$, and $C_{B}$ and $C_{S}=$ non-dimensional coefficients of the DTM, which are dynamically computed from the value of $\mathbf{v}$ at each time step. The modified Leonard tensor $L_{i j}^{m}$ and $L_{i j}^{R}$ in Eq. (5) are defined as

$$
L_{i j}^{R}=\overline{\left(v_{i}-\overline{v_{i}}\right)\left(v_{j}-\overline{v_{j}}\right)}-\overline{\left(v_{i}-\overline{v_{i}}\right)\left(v_{j}-\overline{v_{j}}\right)}
$$

For the motion of the movable structure, the translational velocity $\mathbf{u}^{o b}$ and the angular velocity $\omega^{o b}$ are calculated from the following conservation equations for momentum and angular momentum:

$$
\begin{gathered}
m^{o b} \frac{d u_{i}^{o b}}{d t}=f_{i}^{e x}+f_{i}^{r e} \\
I_{i j}^{o b} \frac{d \omega_{j}^{o b}}{d t}=e_{i j k} r_{j}^{o b}\left(f_{k}^{e x}+f_{k}^{r e}\right)
\end{gathered}
$$

in which $m^{o b}=$ weight of the structure, $\mathbf{I}^{o b}=$ inertia tensor around the center of gravity, $\mathbf{f}^{e x}=$ external force vector, and $\mathbf{f}^{\text {re }}=$ reaction force vector due to contact with the seabed. Assuming a sufficiently small shear force acting on the surface of the structure, the value of $\mathbf{f}^{\text {ex }}$ is calculated from ${ }^{13)}$

$$
f_{\ell}^{e x}=\sum_{i, j, k} F_{o b}\left(-\frac{\partial p}{\partial x_{\ell}}+\rho_{o b} g_{\ell}\right) \Delta x_{i} \Delta y_{j} \Delta z_{k}
$$

in which $\rho_{o b}=$ density of the structure, and $\Delta x_{i}, \Delta y_{j}$, and $\Delta z_{k}=$ widths of numerical cells in the $x, y$, and $z$ directions. When the structure touches the seabed, the value of $\mathbf{f}^{\text {re }}$ is calculated on the assumption ${ }^{14)}$ that the normal component of the relative velocity of the structure at each contact point is zero. 


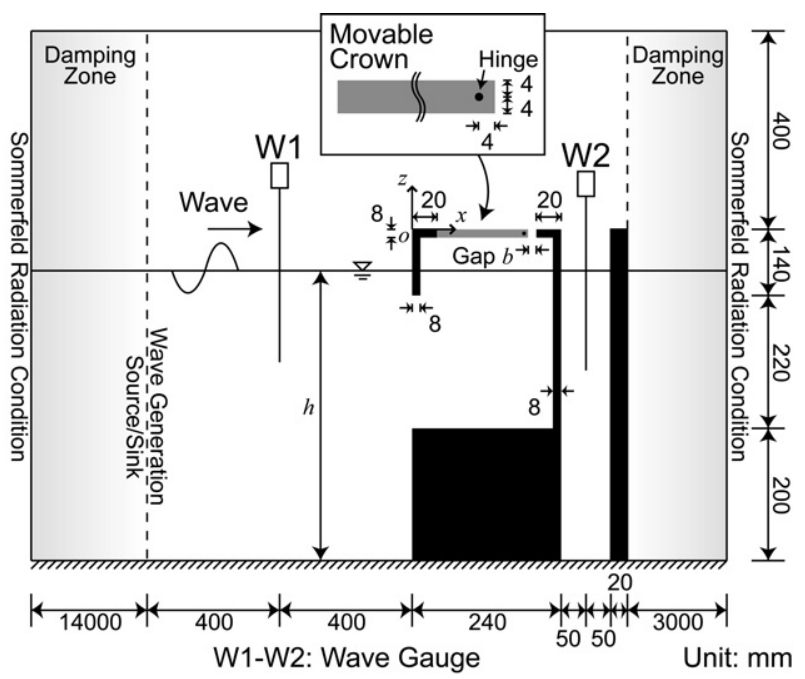

Fig. 2 Schematic of the computational domain.

\section{COMPUTATIONAL DOMAIN AND CONDITIONS}

A schematic of the computational domain modeled from the experimental setup of Kotake et al. ${ }^{3)}$ is shown in Fig. 2. Although the model ${ }^{4)}$ used in this study is a three-dimensional model, a cross-sectional two-dimensional simulation with a unit long-shore distance was performed to reduce the computational time to realistic levels, because fine numerical cells were used, as will be described later in this section.

As shown in Fig. 2, the structure, consisting of a flood-control basin (240 mm wide and $560 \mathrm{~mm}$ high) surrounded by an 8-mm-thick movable crown and an impermeable vertical quay wall, was set in the computational domain, and an impermeable vertical wall (20 mm wide and $560 \mathrm{~mm}$ high) creating a 100-mm-wide wave overtopping pit was set behind the structure. To avoid wave reflection at the seaward and landward boundaries of the computational domain, a 14-m-long damping zone, which coincided with more than two wavelengths of incident regular waves, was attached on the seaward edge, and a 3-mlong damping zone on the landward edge. As illustrated in Fig. 2, the $x$ axis, with an origin at the seaward surface of the structure, was positive landward, and the $z$ axis, with an origin at the top surface of the movable crown, was positive upward.

Figure 3 shows details of the movable crown. In the hydraulic experiment, the hinge of the movable crown was placed $5 \mathrm{~mm}$ from its landward edge when laid flat (angle $\theta=0 \mathrm{rad}$ or $0^{\circ}$ ), as shown in the left-hand figure of Fig. 3. In this condition, there was a 0.75 -mm-wide gap between the edge of the movable crown on the landward side and the impermeable crown. This gap increased concomitantly with an increase in the angle $\theta$, and finally the width of the gap was $1.75 \mathrm{~mm}$ when the crown opened maximally

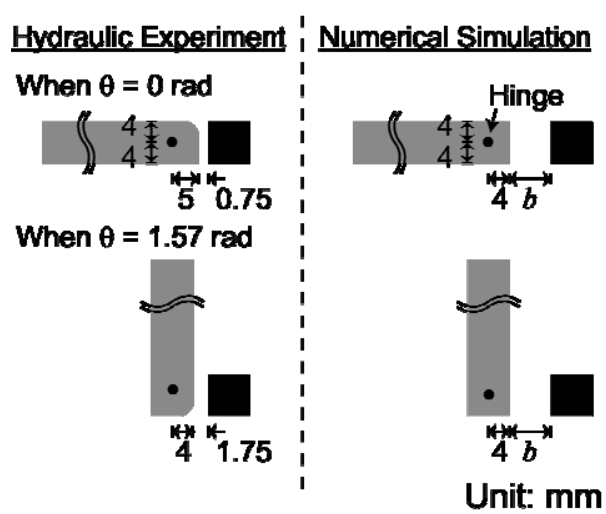

Fig. 3 Modeling of the gap in the movable crown.

Table 1 Regular wave conditions.

\begin{tabular}{ccc}
\hline Still water depth $h[\mathrm{~mm}]$ & Wave period $T[\mathrm{~s}]$ & Wave height $H_{i}[\mathrm{~mm}]$ \\
\hline \multirow{3}{*}{480} & 0.8 & 79 \\
& 1.6 & 97 \\
& 3.2 & 69
\end{tabular}

$\left(\theta=1.57 \mathrm{rad}\right.$ or $\left.90^{\circ}\right)$. However, it was not realistic to use very fine numerical cells that can resolve the changes of $0.75-1.75 \mathrm{~mm}$ associated with the changes in $\theta$. In the numerical simulation, the position of the hinge and the gap were simplified according to the numerical cells, which will be explained later in this section. Specifically, the hinge was positioned $4 \mathrm{~mm}$ away from the landward edge of the movable crown, allowing the width to be $b$ for both $\theta=0 \mathrm{rad}$ and $\theta=1.57 \mathrm{rad}$, as indicated in the right-hand figure of Fig. 3. Although the width of the gap $b$ could be adjusted for consistency of the rate of fluid inflow/ outflow in both the hydraulic experiment and the numerical simulation, it would be difficult to ensure computational accuracy when the gap is represented using one or two numerical cells. In this study, it was assumed that the gap was represented using more than two numerical cells and filled with uniform porous media with a porosity of $m$ and a median grain size of $d_{50}$. Furthermore, the porosity $m$ was assumed to be 0.5 , and the median grain size $d_{50}$ was varied to adjust the rate of fluid inflow/outflow from the gap. Regarding the motion of the movable crown, only Eq. (11) for analyzing the rotational motion of the crown was calculated out of Eqs. (10) and (11), because it was not necessary to consider the translational motion of the movable crown. In Eq. (11), $\mathbf{I}^{o b}, \boldsymbol{\omega}^{o b}$, and $\mathbf{r}^{o b}$ are redefined as inertia tensor around the hinge, angular velocity vector around the hinge, and relative position vector from the hinge. In addition, the value of $\mathbf{f}^{\text {re }}$ was calculated only when the movable crown was flat $(\theta=$ $0 \mathrm{rad})$ or in the maximally vertical position $(\theta=$ $1.57 \mathrm{rad})$. The density of the movable crown was set at $1.19 \times 10^{3} \mathrm{~kg} / \mathrm{m}^{3}$, the same density of the acrylic material used in the hydraulic experiment. It should 


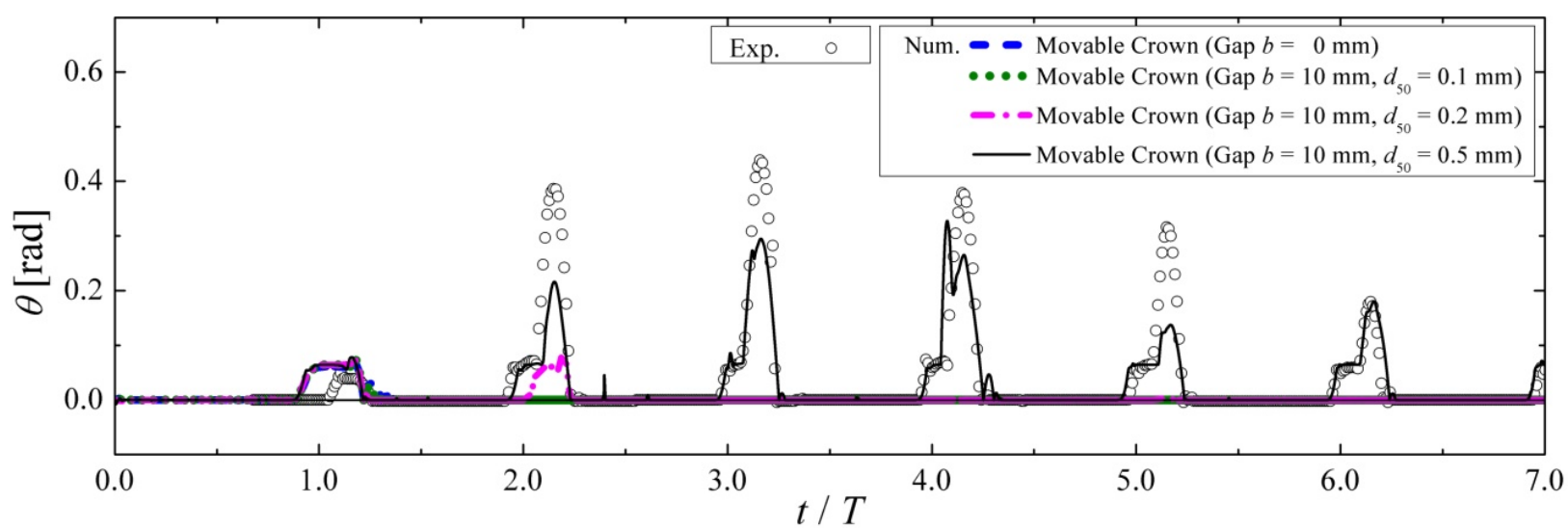

Fig. 4 Comparison of results varying with the angle of the movable crown $\theta\left(T=3.2 \mathrm{~s}, H_{i}=69 \mathrm{~mm}\right)$.

be noted that raised positions of the movable crown can be represented smoothly without resulting in stepped patterns, because the movable crown is expressed using $F_{o b}$, as explained in the previous section.

For the numerical cells, 2-mm square uniform cells, which equaled a quarter of the thickness of the movable crown $(8 \mathrm{~mm})$, were applied to the range of the movable crown movements $(0<x<240 \mathrm{~mm},-10$ $<z<200 \mathrm{~mm}$ ), and non-uniform cells were applied to the rest of the range in order to further reduce the computational time. The following were used for the boundary conditions of flow velocity and pressure: the slip condition for the bottom surface, structure surface, and vertical wall surface; the Sommerfeld radiation condition for the seaward and landward boundaries; and the constant-pressure condition for the top boundary. For the boundary condition of the VOF function, the gradient-free condition was applied to all the boundaries. The initial time step interval was set at $0.005 \mathrm{~s}$, and the time step interval was decreased and increased automatically within its initial value based on the CFL (Courant-FriedrichsLewy) condition. Other parameters, including the gravitational acceleration $g$, were the same as those used in Nakamura and Mizutani ${ }^{4}$.

Although regular waves and tsunamis were employed as incident waves in the hydraulic experiment, three kinds of regular waves (Table 1) and a tsunami were selected in this study. In Table 1, $h, T$, and $H_{i}$ represent the still water depth, wave period, and wave height, respectively. For the regular waves, in order to investigate the difference in the rate of fluid inflow/outflow through the gap, two scenarios were simulated: one with no gap $(b=0 \mathrm{~mm})$ and the other with a gap of five numerical cells $(b=10 \mathrm{~mm})$. In the latter scenario, three different values of the median grain size of the porous media $d_{50}$ were adopted: $0.1 \mathrm{~mm}, 0.2 \mathrm{~mm}$, and $0.5 \mathrm{~mm}$. In addition, a scenario in which the movable crown with $b=0 \mathrm{~mm}$ was fixed at a horizontal position was analyzed to investigate the effect of the rotational motion of the movable crown. For the tsunami, its leading wave was modeled as an increase in the water level in the hydraulic experiment. In this study, a scenario in which an increase rate of the water level was $0.33 \mathrm{~cm} / \mathrm{s}$ on the seaward side of the structure was simulated. For more detailed information on the tsunami, see Kotake et al. ${ }^{3)}$. In this scenario, only the gap of $b=10 \mathrm{~mm}$ and $d_{50}=0.5 \mathrm{~mm}$ was selected because this condition yielded the most reasonable prediction in the scenarios of the regular waves, as will be described in the next section.

\section{NUMERICAL RESULTS AND DISCUSSION}

\section{(1) Wave field around the structure and the be- havior of the movable crown for regular waves}

Figure 4 shows a comparison of the angle of the movable crown $\theta$, in which $t$ represents elapsed time. In Fig. 4, the circles ( $\circ \circ$ ) indicate the results of the hydraulic experiments by Kotake et al. ${ }^{3)}$. The dashed line represents the $b=0 \mathrm{~mm}$ scenario without any gaps, and the dotted, dashed-dotted, and solid lines represent the $b=10 \mathrm{~mm}$ scenarios for different porous media of $d_{50}=0.1 \mathrm{~mm}, 0.2 \mathrm{~mm}$, and $0.5 \mathrm{~mm}$, respectively. As shown in Fig. 4, when the movable crown was set at $b=0 \mathrm{~mm}$ (dashed line) and $b=$ $10 \mathrm{~mm}$ with the porous media of $d_{50}=0.1 \mathrm{~mm}$ (dotted line), the movable crown opened only once, failing to predict the experimental results. Figure 5 shows the wave field around the structure and the behavior of the movable crown when the width of the gap $b$ was set at $0 \mathrm{~mm}$. In the figure, $p^{\prime}$ represents pressure variation. Figure 5(a) shows that the movable crown was pushed up slightly by the air trapped in the flood-control basin when the water level was high. At this moment, the increase in the water level in the flood-control basin was slower 

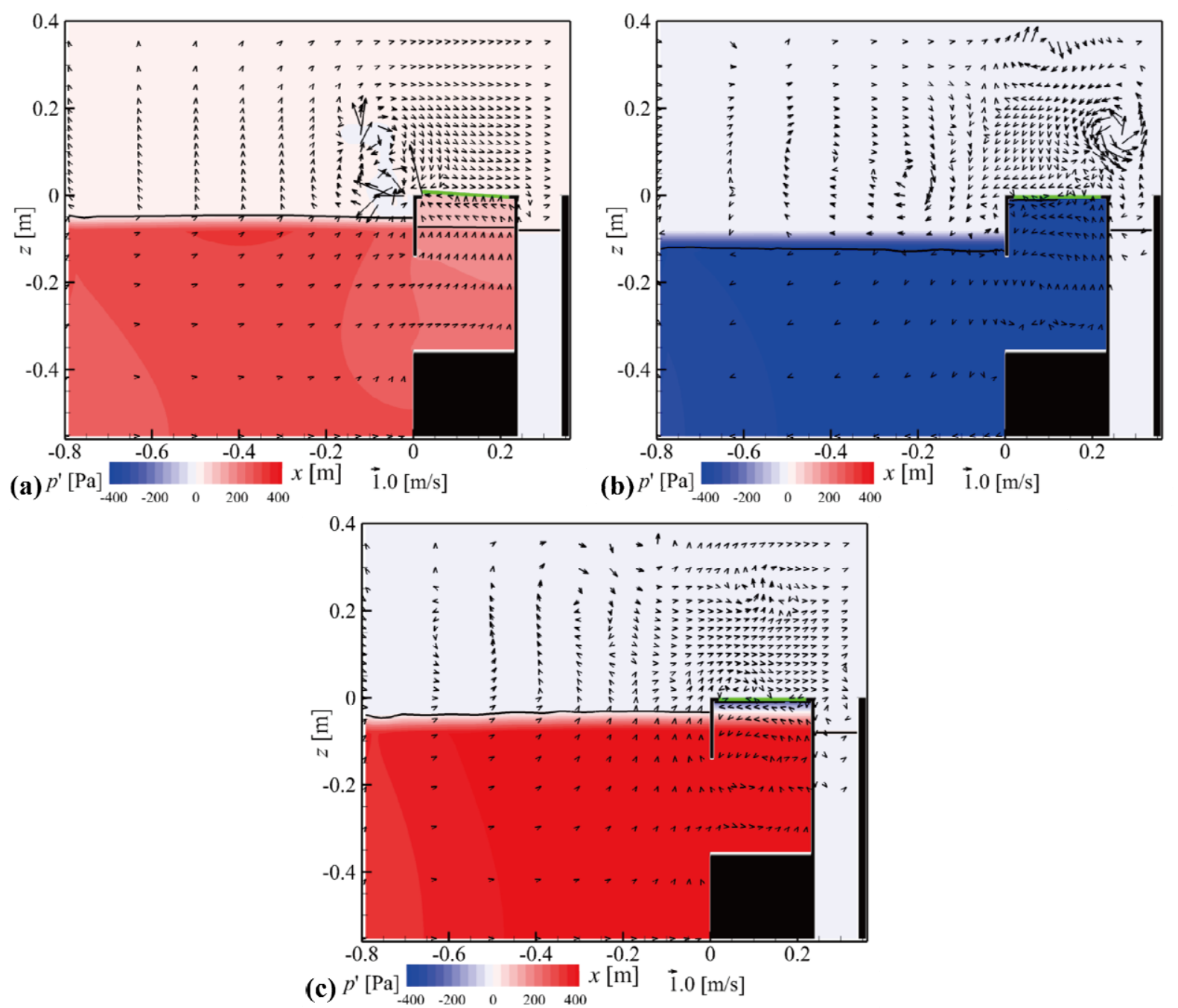

Fig. 5 Wave field around the structure and the behavior of the movable crown with $b=0 \mathrm{~mm}$ ( $T=3.2 \mathrm{~s}, H_{i}=69 \mathrm{~mm}$ ): (a) $t / T=1.0$; (b) $t / T=1.5$; and (c) $t / T=2.0$.

than that in front of the structure. However, the pressure underneath the movable crown in the flood-control basin increased due to the raised pressure associated with the increased water level in front of the structure, and so raised the movable crown. After that, Fig. 5(b) shows that the flood-control basin was filled with water even at the time of backwash, because there was no gap for air to enter once the movable crown was closed. As shown in Fig. 5(c), the movable crown did not open even when the water level increased again, because there was no air in the flood-control basin. Similar tendencies were observed for the movable crown with $b=10 \mathrm{~mm}$ and $d_{50}=0.2 \mathrm{~mm}$ (dashed-dotted line in Fig. 4). Although this condition allowed a slightly easier air inflow, the crown opened only twice. Furthermore, although the crown opened a second time up to a degree of $0.06 \mathrm{rad}$, it did not clearly repeat the movement, suggesting an underestimation of the experimental results. On the other hand, for $b$ $=10 \mathrm{~mm}$ and $d_{50}=0.5 \mathrm{~mm}$ (solid line in Fig. 4), the movable crown opened up to approximately
$0.06 \mathrm{rad}$, and was followed by greater upward movement, which agreed reasonably well with the experimental results. For this case, the wave field around the structure and the behavior of the movable crown are shown in Fig. 6. As seen in Fig. 6(a), the air trapped in the flood-control basin pushed up the movable crown slightly, and the air was expelled from the gap. The movable crown was then raised more significantly by the increased water level in the flood-control basin, and some of the water was discharged from the gap to the seaward side (Fig. 6(b)). As indicated in Fig. 4, although the maximum values of $\theta$ were slightly underestimated compared to the experimental results, they fluctuated depending on the different wave conditions in the flood-control basin, which suggests similarities with the experimental results.

Figures $\mathbf{7}$ and $\mathbf{8}$ compare the water level changes $\eta$ in front of the structure (W1 in Fig. 2). For $T=$ $0.8 \mathrm{~s}$, as shown in Fig. 7, no obvious difference was observed among the different conditions of the crown and the gap, suggesting reasonable agreement 

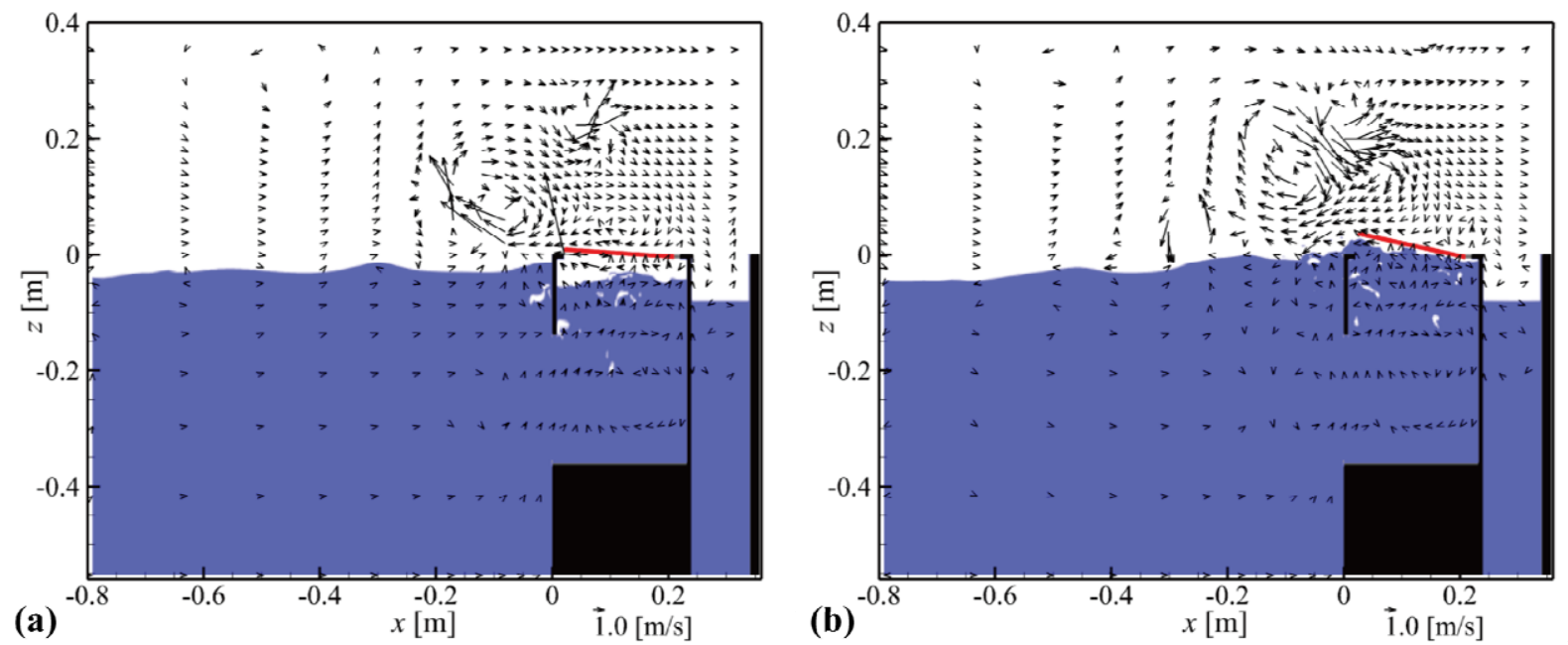

Fig. 6 Wave field around the structure and the behavior of the movable crown with $b=10 \mathrm{~mm}$ and $d_{50}=0.5 \mathrm{~mm}$ ( $T=3.2 \mathrm{~s}, H_{i}=69 \mathrm{~mm}$ ); (a) the movement by air pressure; and (b) the movement by the waves.

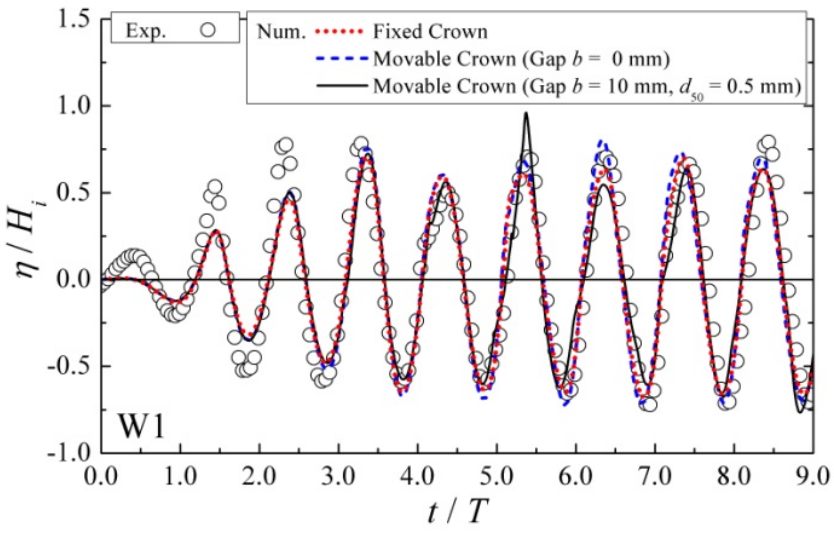

Fig. 7 Comparison of the water level changes $\eta$ in front of the structure (W1; $T=0.8 \mathrm{~s}, H_{i}=79 \mathrm{~mm}$ ).

with the experimental results. This agreement includes the fluctuations of the amplitude of $\eta$ after the fourth wave $(t / T>3.0)$, when the wave field was sufficiently stable, although there were slight differences at around $t / T=5.4$ and 6.4. On the other hand, for $T=3.2 \mathrm{~s}$, Fig. 8 shows a small difference between the fixed crown (dotted line) and the movable crown with $b=0 \mathrm{~mm}$ (dashed line), suggesting an overestimation of the experimental results in terms of the amplitude of $\eta$. Compared with these two scenarios, the amplitude of $\eta$ for the movable crown with $b=10 \mathrm{~mm}$ and $d_{50}=0.5 \mathrm{~mm}$ (solid line) is slightly smaller, suggesting a similarity with the experimental results. However, the troughs still tended to be slightly overestimated after the third wave $(t / T>2.0)$, when the wave field was sufficiently stable. Based on the observation of peaks and troughs, the wave shapes seem uneven, suggesting a small disturbance in the water level. This tendency is more obvious from the numerical results, although this is also observed in the experimental data. The small disturbance in the peaks is due to the air flow from the opened movable crown, while the small

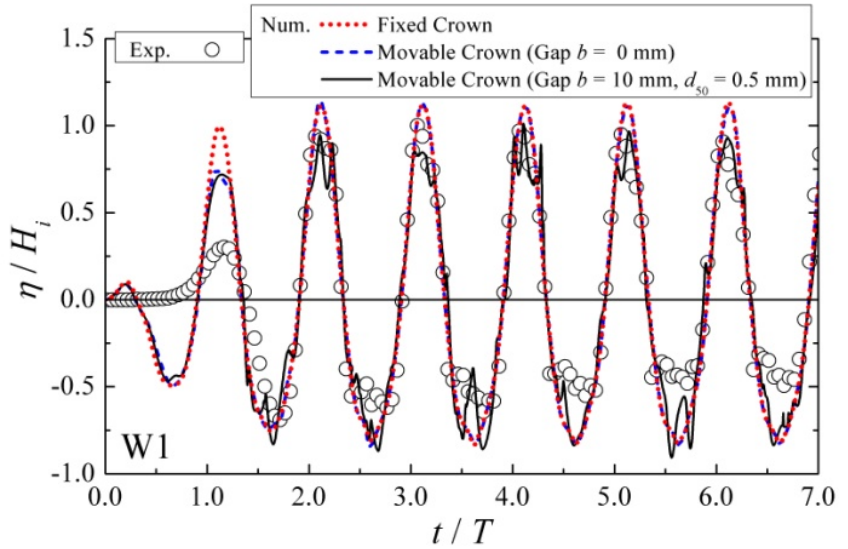

Fig. 8 Comparison of the water level changes $\eta$ in front of the structure (W1; $T=3.2 \mathrm{~s}, H_{i}=69 \mathrm{~mm}$ ).

disturbance in the troughs is due to air bubbles created by air flow entering the flood-control basin from the gap in the crown. The overestimation of the troughs can be explained by the influence of air bubbles on the waves. In this scenario, the reflection coefficient was observed to be between 0.8 and 0.9 in the hydraulic experiment. In order to investigate the mechanism of the lowered reflection coefficient, Fig. 9 shows the distributions of the average water level, average flow velocity, and average vorticity $\bar{\omega}$ around the structure for the same scenario as Fig. 8. Each average value was calculated from the numerical results during the two wave periods from $t / T=$ 4.0 to $t / T=6.0$, and the positive $\bar{\omega}$ indicates counterclockwise vortices. In Fig. 9, the average water level represents contour lines with the average VOF function of 0.5. In order to derive the average vorticity in the water phase, the products of vorticity and VOF function were calculated at each time, and their average was defined as $\bar{\omega}$. As seen in Fig. 9, there was no major difference in the distribution of $\bar{\omega}$ between the fixed crown (Fig. 9(a)) and the movable crown with $b=0 \mathrm{~mm}$ (Fig. 9(b)). For the 

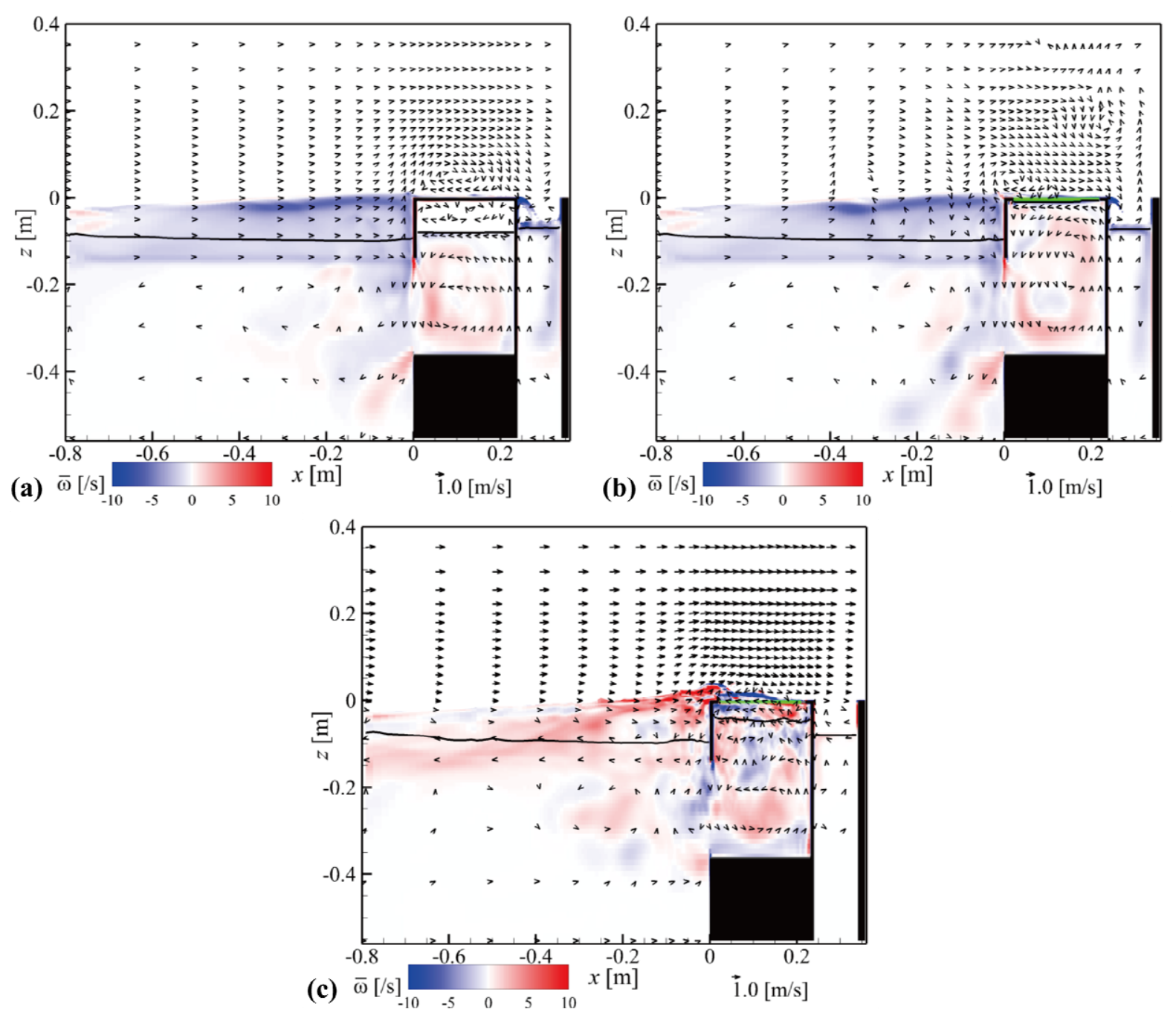

Fig. 9 Distribution of the average water level, average flow velocity, and average vorticity $\bar{\omega}$ around the structure $\left(T=3.2 \mathrm{~s}, H_{i}=69\right.$ $\mathrm{mm}$ ); (a) the fixed crown; (b) the movable crown with $b=0 \mathrm{~mm}$; and (c) the movable crown with $b=10 \mathrm{~mm}$ and $d_{50}=0.5 \mathrm{~mm}$.

movable crown with $b=0 \mathrm{~mm}$, as explained earlier, the crown opened only once at the beginning despite its movable state. This suggests that the wave energy dissipated by the rotational motion was relatively small, resulting in the small difference between the fixed crown and the movable crown with $b=0 \mathrm{~mm}$. A similar explanation seems to apply to Fig. 8, which shows no significant difference in the water level changes $\eta$. On the other hand, for $b=10 \mathrm{~mm}$ and $d_{50}=0.5 \mathrm{~mm}$, Fig. 9(c) shows that although the distribution of $\bar{\omega}$ in front of the structure $(x<0 \mathrm{~mm})$ slightly differs from those in Figs. 9(a) and (b), there was no major difference in absolute values between the three scenarios. A similar tendency is also observed for $\bar{\omega}$ below the still water surface in the flood-control basin $(0<x<240 \mathrm{~mm}, \mathrm{z}<-80 \mathrm{~mm})$. However, a greater dissipation of the wave energy was inferred from the greater absolute value of $\bar{\omega}$ above the still water surface in the flood-control basin and above the structure, because there was greater inflow and outflow of fluid though the gap in the crown due to the increased motion of the movable crown, as mentioned earlier. In addition, it was inferred from Fig. 4 that the wave energy dissipation associated with the motion of the movable crown was greater than that for the other scenarios. Consequently, the wave energy was dissipated effectively, which contributed to lowering the amplitude of $\eta$ (Fig. 8).

Finally, the water level changes $\eta$ in the wave overtopping pit (W2 in Fig. 2) are compared in Figs. 10 and 11. For $T=0.8 \mathrm{~s}$, Fig. 10 shows that although slight fluctuations were observed for the fixed crown (dotted line), a small amount of wave overtopping predicted in the numerical simulation was in agreement with the experimental data (circles). Similar to Fig. 8, for $T=3.2 \mathrm{~s}$, Fig. 11 indicates that there was no significant difference between the fixed crown (dotted line) and the movable crown with $b=0 \mathrm{~mm}$ (dashed line), which suggested an overestimation of the experimental results. As shown in Fig. 8, the water level changes $\eta$ in front of the structure, particularly the wave peaks, were overestimated. In addition, as shown in Fig. 4, the 


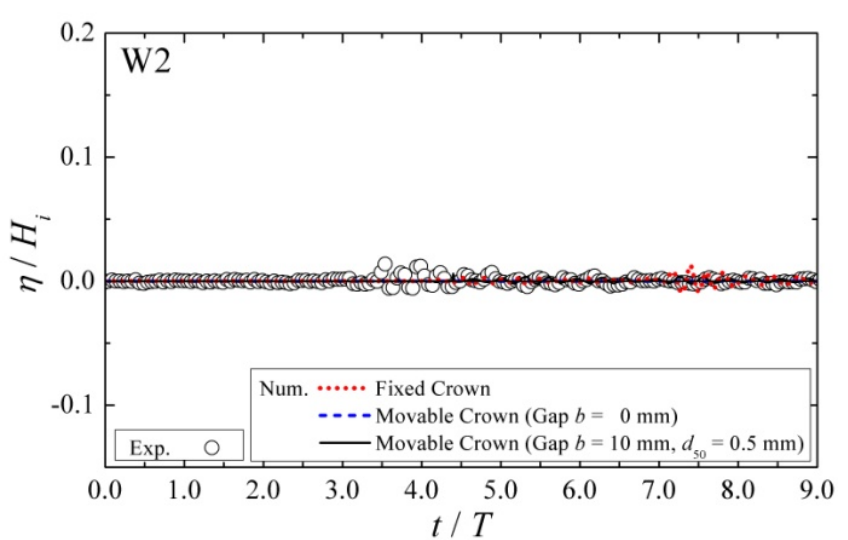

Fig. 10 Comparison of the water level changes $\eta$ in the wave overtopping pit (W2; $T=0.8 \mathrm{~s}, H_{i}=79 \mathrm{~mm}$ ).

crown did not seem to provide a lowering effect against wave overtopping because of its immobility, except just before $t / T=2.0$ when the crown opened only once. For these two reasons, the increase in the water level in the wave overtopping pit was overestimated. Conversely, Fig. 11 suggests that the numerical results for $b=10 \mathrm{~mm}$ and $d_{50}=0.5 \mathrm{~mm}$ (solid line) underestimated the experimental data. In this scenario, the wave peaks were generally consistent (Fig. 8). Furthermore, the overall movement of the movable crown was captured reasonably well, although the maximum values of $\theta$ were slightly underestimated (Fig. 4). Despite this, the increase in the water level in the wave overtopping pit was underestimated, as described earlier. This unconformity can be explained by the different wave overtopping rates through the gap. In the numerical simulation, the width of the gap decreases gradually as the angle of the movable crown $\theta$ increases, showing the smallest gap at $\theta=0.79 \mathrm{rad}\left(45^{\circ}\right)$. On the other hand, in the hydraulic experiment, the gap did not decrease as much because the landward surface of the movable crown was curved, possibly resulting in the underestimation of wave overtopping from the gap. This also suggests greater wave overtopping rates in the hydraulic experiment. Consequently, it is essential to provide countermeasures to mitigate wave overtopping from the gap in this structure.

As described so far, the predictive capability of the model was demonstrated in terms of the wave field around the structure and the behavior of the movable crown. In addition, the mechanisms of the movable crown behavior and wave energy dissipation were revealed from the analysis of the numerical results. Specifically, it was found that the motion of the movable crown was affected by the air trapped in the flood-control basin at the time of uprush and the air flow entering the flood-control basin at the time of backwash. Furthermore, it was demonstrated that the rate of fluid inflow/outflow through the gap is an

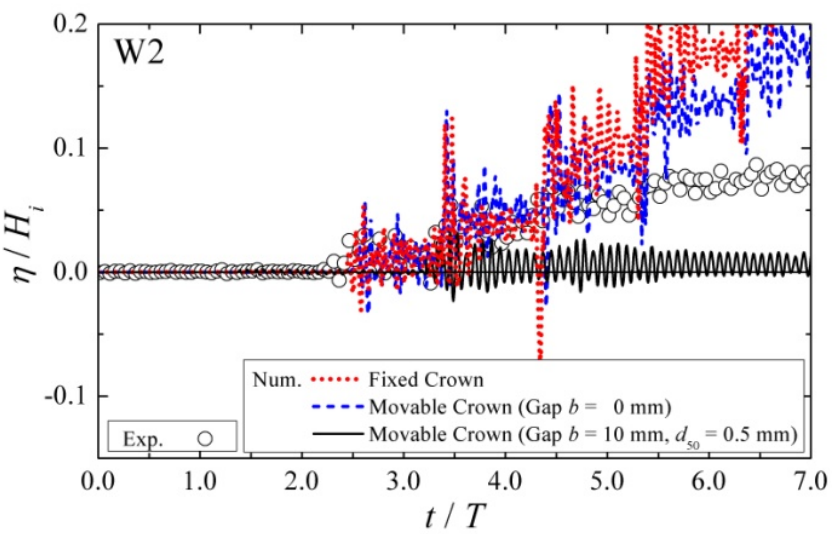

Fig. 11 Comparison of the water level changes $\eta$ in the wave overtopping pit (W2; $T=3.2 \mathrm{~s}, H_{i}=69 \mathrm{~mm}$ ).

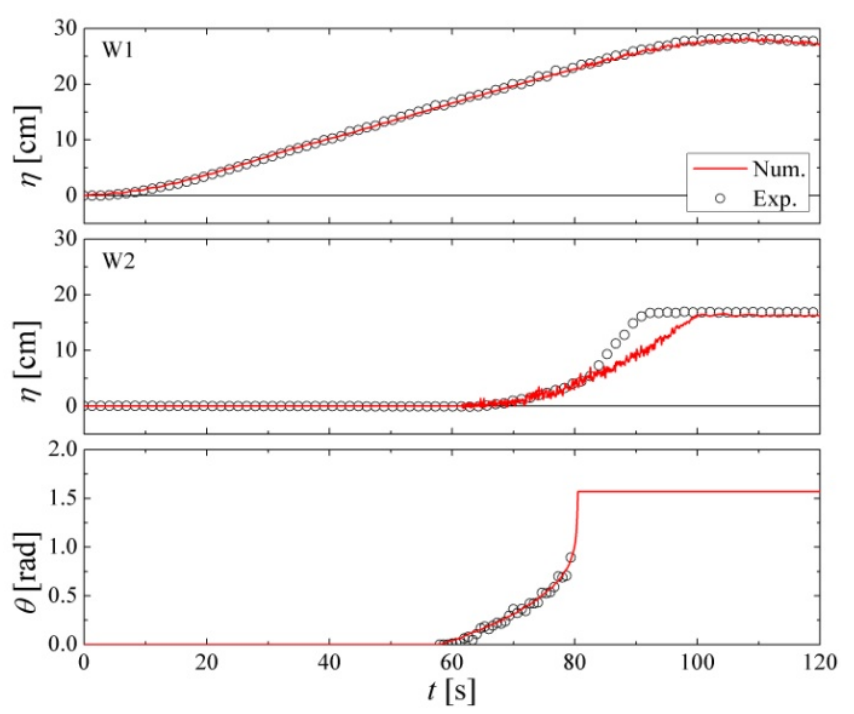

Fig. 12 Comparison of the water level changes $\eta$ in the front of the structure (W1), the water level changes $\eta$ in wave overtopping pit (W2), and the angle of the movable crown $\theta$ during the tsunami attack ( $\left.b=10 \mathrm{~mm}, d_{50}=0.5 \mathrm{~mm}\right)$.

important parameter, because it influenced not only the movable crown behavior and wave overtopping rates, but also the wave field in front of the structure. As a result, it is verified that the model is a useful tool in designing the structure, because it allows appropriate evaluation of the performance of the structure if conformity with the hydraulic experiment is achieved for the fluid inflow/outflow rate.

\section{(2) Wave field around the structure and the be- havior of the movable crown for tsunamis}

Figure 12 shows a comparison of the water level changes $\eta$ in front of the structure (W1 in Fig. 2), those in the wave overtopping pit (W2 in Fig. 2), and the angle of the movable crown $\theta$. In the figure, the circles represent the experimental data, and the solid lines represent the numerical results for the scenario of $b=10 \mathrm{~mm}$ and $d_{50}=0.5 \mathrm{~mm}$, which was selected because of the conformity of the results for the regular waves. It should be noted that the experimental values of $\theta$ are presented up to $T=80 \mathrm{~s}$ because of 

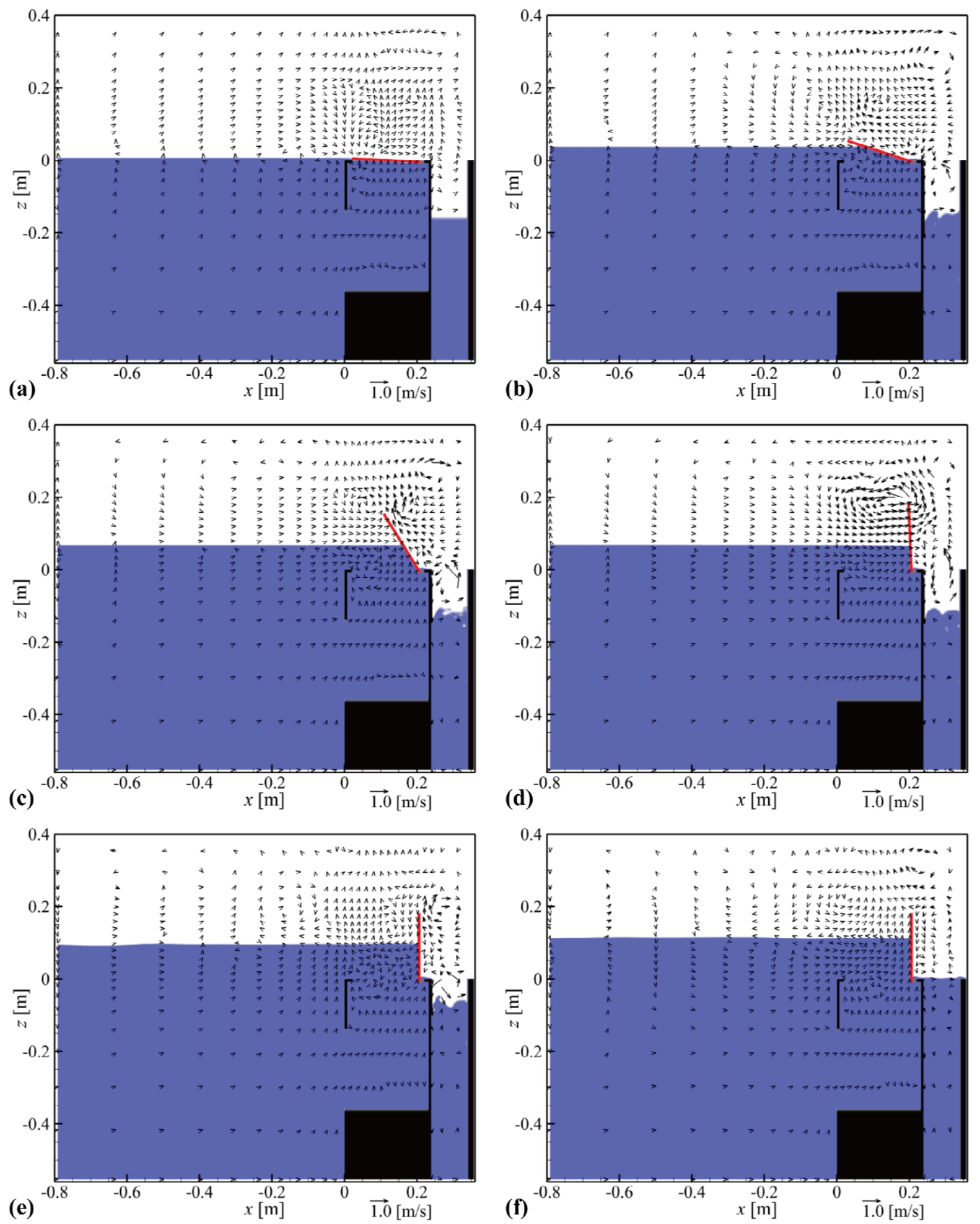

Fig. 13 Wave field around the structure and the behavior of the movable crown during the simulated tsunami attack $(b=10 \mathrm{~mm}$, $d_{50}=0.5 \mathrm{~mm}$ ); (a) $t=60.0 \mathrm{~s}$; (b) $t=70.0 \mathrm{~s}$; (c) $t=80.0 \mathrm{~s}$; (d) $t=80.5 \mathrm{~s} ;$ (e) $t=90.0 \mathrm{~s}$; and (f) $t=100.0 \mathrm{~s}$.

the limitation of the experimental setup.

As shown in Fig. 12, the numerical model predicts well the elevation of the water level in front of the structure (W1 in Fig. 2). The angle of the movable crown $\theta$ increased slowly for approximately $20 \mathrm{~s}$ up to $\theta=0.8 \mathrm{rad}$, and then increased rapidly up to the maximum angle $\left(\theta=1.57 \mathrm{rad}\right.$ or $\left.90^{\circ}\right)$, which was in good agreement with the experimental results. For this case, the wave field around the structure and the behavior of the movable crown are shown in Fig. 13. As seen in Fig. 13(a), the movable crown begins to increase as the water level in the flood-control basin reaches up underneath the crown. After that, the angle of the movable crown $\theta$ increases gradually as the water level increases, resulting in the disturbance of the water surface in the wave overtopping pit due 
to wave overtopping from the gap (Figs. 13(b) and (c)). From $t=80.0 \mathrm{~s}$ to $t=80.5 \mathrm{~s}$, the movable crown rose rapidly (Fig. 13(d)). Subsequently, continuous wave overtopping was observed with the movable crown raised at the maximum angle (Fig. 13(e)), and finally the wave overtopping pit was filled with water at $t=100 \mathrm{~s}$ (Fig. 13(f)). Figure 12 also shows that the elevation of the water level in the wave overtopping pit (W2 in Fig. 2) was well predicted up to shortly after $t=80 \mathrm{~s}$. However, the subsequent elevation of the water level was underestimated. Specifically, in the hydraulic experiment, the rising rate of the water surface increased gradually, and finally the water surface reached the full level of the wave overtopping pit $(\eta=16.0 \mathrm{~cm})$ at $t=90 \mathrm{~s}$. In the numerical simulation, the water level continued to increase at almost the same rising rate. As mentioned earlier, the width of the gap $b$ was increased from $0.75 \mathrm{~mm}$ to $1.75 \mathrm{~mm}$ with an increase in the angle of the movable crown $\theta$ in the hydraulic experiment, whereas the width of the gap $b$ decreases from $10 \mathrm{~mm}$ to $8.4 \mathrm{~mm}$ at angles of $\theta=0$ rad to $\theta=$ $0.79 \mathrm{rad}$, and conversely increases from $8.4 \mathrm{~mm}$ to $10 \mathrm{~mm}$ at angles of $\theta=0.79 \mathrm{rad}$ to $\theta=1.57 \mathrm{rad}$. This difference led to an underestimation of the fluid inflow/outflow rate, resulting in the underestimation of the water surface elevation in the wave overtopping pit.

From these results, it was demonstrated that the numerical model can be applied to tsunamis as well as regular waves in designing the structure. However, the results also suggest that the relation between the fluid inflow/outflow rate through the gap and the angle of the movable crown $\theta$ in the numerical simulation should be identical to that in the hydraulic experiment particularly when analyzing the wave overtopping rates behind the structure in detail, including the delay time of the onset of inundation.

\section{CONCLUSIONS}

In this study, a three-dimensional coupled fluidstructure-sediment interaction model ${ }^{4)}$, which has the computational capability for analyzing air-waterstructure interactions, was applied to a hydraulic experiment $^{3)}$ of a tsunami/storm-surge mitigation structure attached with a movable crown. To demonstrate the predictive capability of the model, numerical results computed using the model were analyzed and compared with experimental data in terms of the water level changes around the structure and the behavior of the movable crown. Furthermore, numerical results were further analyzed and dis- cussed to investigate the mechanisms of the movable crown behavior and wave energy dissipation. The main conclusions from this study are summarized below:

1. The relation between the fluid inflow/outflow rate through the gap in the crown and the angle of the movable crown is an important parameter in predicting the wave field around the structure and the behavior of the movable crown. This suggests that the parameter in the numerical simulation should be identical to that in the hydraulic model when conducting a numerical simulation of other types of structures significantly affected by fluid inflow/outflow rates from gaps.

2. The influence of the air behavior is of significance for this structure. Specifically, it was observed that the movable crown was slightly raised by the air trapped in the flood-control basin, and then raised further by an increase in the water level in the flood-control basin. In addition, it was observed that the behavior of the movable crown was influenced by the air flow entering the flood-control basin from the gap at the time of backwash.

3. The structure has the mechanism of effective wave energy dissipation by the motion of the movable crown and vortices forming above the still water surface in the flood-control basin and above the structure.

However, this study revealed new challenges in that the computational capability of the model is somewhat unsatisfactory in predicting the water level changes in the wave overtopping pit and the angle of the movable crown with high accuracy. In further investigation, it is essential to obtain conformity in the parameter for both the hydraulic experiment and the numerical simulation.

\section{REFERENCES}

1) Shuto, N., Imamura, F., Koshimura, S., Satake, K. and Matsutomi, H.: Encyclopedia of Tsunami, Asakura Publishing Co., Ltd., 350 p., 2007 (in Japanese).

2) Ministry of Agriculture, Forestry and Fisheries (MAFF) and Ministry of Land, Infrastructure, Transport and Tourism (MLIT): Maintenance Manual of Coastal Facilities for Life-Cycle Management - Inspection and Diagnosis of Dikes, Bulkhead, and Seawall - (tentative), 105 p., 2008 (in Japanese).

3) Kotake, Y., Kanazawa, T., Yamazaki, K. and Matsumura, A.: Development of disaster mitigative caisson with retractable seawall on the crown, J. JSCE, Ser. B3 (Ocean Eng.), Vol. 67, No. 2, pp. I_631-I_636, 2011 (in Japanese).

4) Nakamura, T. and Mizutani, N.: Numerical simulation of local scouring around inland structure due to run-up tsunami using three-dimensional coupled fluid-structuresediment interaction model, Proc., 24th CFD Symposium, 
JSFM, E10-4, 9 p., 2010 (in Japanese).

5) Kunugi, T.: MARS for multiphase calculation, CFD J., No. 9, Vol. 1, IX-563, 1-10, 2000.

6) Kajishima, T., Takiguchi, S., Hamasaki, H. and Miyake, Y.: Turbulence structure of particle-laden flow in a vertical plane channel due to vortex shedding, JSME Int. J. Ser. B, Fluids and Thermal Eng., Vol. 44, No. 4, pp. 526-535, 2001.

7) Nakamura, T., Mizutani, N. and Koike, T.: Discussion on fluid-structure coupled numerical simulation using body force type of immersed boundary method, Proc., 63rd JSCE Annual Meeting, JSCE, II-193, pp. 385-386, 2008 (in Japanese).

8) Mizutani, N., McDougal, W. G. and Mostafa, A. M.: BEMFEM combined analysis of nonlinear interaction between wave and submerged breakwater, Proc., 25th Int. Conf. on Coastal Eng., ASCE, pp. 2377-2390, 1996.

9) Brackbill, J. U., Kothe, B. D. and Zemach, C.: A continuum method for modeling surface tension, J. Comp. Phys., Vol. 100, pp. 335-354, 1992.
10) Horiuti, K.: A new dynamic two-parameter mixed model for large-eddy simulation, Phys. Fluids, Vol. 9, No. 11, pp. 3443-3464, 1997.

11) Kawasaki, K.: Numerical simulation of breaking and post-breaking wave deformation process around a submerged breakwater, Coastal Eng. J., JSCE, Vol. 41, No. 3-4, pp. 201-223, 1999.

12) Coastal Development Institute of Technology (CDIT): Research and Development of Numerical Wave Flume (Super Roller Flume for Computer Aided Design of Maritime Structure), CDIT, 296 p., 2001 (in Japanese).

13) Xiao, F., Yabe, T., Ito, T. and Tajima, M.: An algorithm for simulating solid objects suspended in stratified flow, Comp. Phys. Communications, Vol. 102, pp. 147-160, 1997.

14) Honda, K., Tomita, T., Nishimura, D. and Sakaguchi, A.: Numerical modeling of tsunami drifted bodies, Annual $J$. Civil Eng. in Ocean, Vol. 25, pp. 39-44, 2009 (in Japanese).

(Received October 10, 2012) 